\section{Los vicios de nulidad y su incidencia en la gestión de los procedimientos administrativos disciplinarios en el año 2019}

\author{
The vices of nullity and their impact on the management of \\ the procedures administrative disciplinary in 2019
}

\begin{abstract}
RESUMEN
El presente artículo está orientado a explicar y dar a conocer la incidencia que tienen los vicios de nulidad en la gestión de los procedimientos administrativos disciplinarios desarrollados por las entidades públicas en el año 2019, como parte del ejercicio de su potestad disciplinaria a fin de garantizar el adecuado funcionamiento de la Administración Pública. En este artículo se analiza la responsabilidad administrativa disciplinaria que alcanza a los servidores públicos en el ejercicio de sus funciones cuando incurren en hechos que resultan contrarios al ordenamiento jurídico, lo que genera que se les juzgue mediante un procedimiento administrativo disciplinario a fin de imponerles una sanción. Sin embargo, en el desarrollo de dicho procedimiento administrativo disciplinario es frecuente que se produzcan vicios que acarrean la nulidad de los referidos procedimientos. En ese sentido, el análisis del presente artículo se realizó sobre la base de 50 servidores de distintas entidades públicas, quienes fueron encuestados, habiéndose llegado a determinar como resultado algunos factores que dan lugar a la producción de vicios de nulidad, así como las consecuencias nocivas que dichos vicios generan para la adecuada gestión de los procedimientos administrativos disciplinarios.
\end{abstract}

Palabras clave: Potestad disciplinaria; procedimientos administrativos disciplinarios; vicios de nulidad; responsabilidad administrativa disciplinaria.

\begin{abstract}
This article is oriented to explain and publicize the incidence the vices of nullity in the management of the procedures administrative disciplinary developed by public entities in 2019, as part of the exercise of their power disciplinary in order to guarantee the proper functioning of the Public Administration. This article analyzes responsibility administrative disciplinary that reaches to servants public in the exercise of their functions when they incur in acts that are contrary
\end{abstract}

Eliana Sthefanie Torres Morón

estm29@gmail.com

Autoridad Nacional del Servicio Civil - SERVIR. Lima, Perú

Presentado: 17/02/2021 - Aceptado: 01/03/2021 - Publicado: 17/06/2021

(C) Los autores. Este artículo es publicado por la revista Gestión en el Tercer Milenio de la Facultad de Ciencias Administrativas Universidad Nacional Mayor de San Marcos. Este es un artículo de acceso abierto, distribuido bajo los términos de la licencia Creative Commons Atribución 4.0 Internacional (CC BY 4.0) [https://creativecommons.org/licenses/by/4.0/deed.es] que permite el uso, distribución y reproducción en cualquier medio, siempre que la obra original sea debidamente citada de su fuente original. 
to the legal system, what causes them to be judged through an procedure administrative disciplinary in order to impose a penalty. However, in the development of said procedure administrative disciplinary, it is frequent that vices occur that lead to the invalidity of the referred procedures. In this sense, the analysis of this article was carried out on the basis of 50 servers from different entities public, who were surveyed, having come to determine as a result some factors that give rise to the production of vices nullity, as well as the consequences harmful that these vices generate for the proper management of procedures disciplinary administrative.

Keywords: Power disciplinary; procedures administrative disciplinary; vices of nullity; and responsibility administrative disciplinary.

\section{INTRODUCCIÓN}

Los servidores públicos forman parte de la Administración Pública, lo que implica que para ellos la potestad sancionadora del Estado no es aquella que se dirige contra cualquier ciudadano que incurra en alguna conducta infractora, sino que tiene carácter especial pues se dirige precisamente hacia las personas que actúan en nombre y representación del Estado. Si bien tanto los particulares como los servidores públicos pueden ser objeto de la potestad sancionadora del Estado, solo estos últimos pueden ser objeto de la potestad disciplinaria del Estado, en virtud al interés legítimo con que este cuenta para sancionar a las personas que actúan en su nombre y representación.

Respecto a la potestad disciplinaria Marina (2006) sostiene lo siguiente:

Sirve a la Administración para la tutela de su organización, de forma que ésta pueda cumplir su función de servicio público eficaz, imparcial y con respeto a la legalidad. El poder disciplinario es, por tanto, un instrumento del que dispone la Administración para luchar contra los funcionarios que incumplen o abusan de sus funciones. (p. 44)

En esa línea, para que las entidades públicas puedan ejercer su potestad disciplinaria necesitan de un instrumento, un medio, a través del cual puedan desplegar tal potestad. Dicho instrumento o medio se representa en el procedimiento administrativo disciplinario (PAD), entendido este como el conjunto de actuaciones tendientes a determinar si alcanza o no responsabilidad administrativa disciplinaria a un servidor público y, como consecuencia de ello, si corresponde o no imponer una sanción determinada.

El procedimiento administrativo disciplinario se presenta entonces como el mecanismo a través del cual las entidades públicas pueden ejercer su potestad disciplinaria para determinar si alcanza o no responsabilidad administrativa disciplinaria a un servidor público; $y$, al mismo tiempo, constituye una garantía para el servidor público, habida cuenta que el procedimiento le va permitir conocer los cargos que se le imputan, ejercer su derecho de defensa, exponer alegatos, ofrecer pruebas, impugnar la decisión de primera instancia, entre otros derechos.

Con tal propósito, la Ley N 30057 - Ley del Servicio Civil y su Reglamento General aprobado por Decreto Supremo N 040-2014-PCM, han regulado el procedimiento administrativo disciplinario aplicable a los servidores públicos, el cual se encuentra conformado por una serie de actuaciones que, a su vez, forman parte de una etapa determinada. Las etapas comprendidas en el procedimiento administrativo disciplinario formalmente iniciado son: a) la etapa de instrucción y b) la etapa de sanción; asimismo, hay una etapa previa al inicio formal del procedimiento conocida como la etapa de indagaciones preliminares o precalificación.

Tales etapas se encuentran sujetas, por un lado, a principios que determinan su validez y por otro, a determinadas garantías inherentes a un debido procedimiento. Es así que, cuando se produce la inobservancia de algún principio o de alguna garantía inherente al debido procedimiento, se configura un vicio en el desarrollo de los procedimientos administrativos disciplinarios, que trae como consecuencia la declaratoria 
de nulidad del procedimiento hasta el momento en que se produjo el vicio de nulidad.

En ese sentido, existe una situación problemática referida a los errores que se cometen en el desarrollo de los procedimientos administrativos disciplinarios, ya sea por la transgresión de algunos principios o por la transgresión de alguna garantía inherente al debido procedimiento, lo que vicia de nulidad los procedimientos generando que estos tengan que anularse y retrotraerse para después tener que volver a desarrollarse.

Tal situación problemática afecta no solo a las entidades públicas que tienen que utilizar doblemente sus recursos humanos y logísticos para desarrollar más de una vez un mismo procedimiento, sino también a los servidores públicos habida cuenta que tienen que soportar la carga de ser procesados por un tiempo que excede el legalmente permitido, dejándolos en situación de incertidumbre. Sobre la problemática advertida, Morales (2020) sostiene lo siguiente:

Yendo a la estadística, el porcentaje de resoluciones anuladas o revocadas por las Salas del Tribunal del Servicio Civil por las razones señaladas representa aproximadamente el 38\% del total de emitidas, lo que pone en evidencia la insuficiencia del análisis jurídico del Procedimiento Administrativo Disciplinario realizado por las autoridades previo a la elevación de los recursos de apelación. (p. 58)

En ese contexto, la situación problemática materia del presente artículo se encuentra referida a los vicios de nulidad y su incidencia en la gestión de los procedimientos administrativos disciplinarios llevados a cabo por las entidades públicas en el año 2019.

\section{OBJETIVO DEL ARTÍCULO}

El tema abordado en el presente artículo tiene como objetivo dar a conocer que la escasa capacitación recibida sobre régimen disciplinario incide en la producción de vicios de nulidad; así como que la declaración de nulidad de los procedimientos administrativos disciplinarios incide en la prescripción de dichos procedimientos y en la interposición de demandas de indemnización por la ejecución de sanciones declaradas nulas.

A fin de lograr tal objetivo, la técnica de recolección de datos utilizada es la encuesta, la cual ha sido sometida a un juicio de validez por parte de expertos en la materia, habiendo obtenido como resultado que el $100 \%$ de los ítems fueron validados; asimismo, dicha encuesta pasó un análisis de confiabilidad. El procedimiento de selección de la muestra es no probabilístico de muestreo por conveniencia, en base al cual, según Tamayo (2001) "se seleccionan las unidades muestrales de acuerdo a la conveniencia o accesibilidad del investigador" (p. 13). Tomando en cuenta el procedimiento de selección de la muestra, así como la accesibilidad del investigador, se ha considerado conveniente una muestra de 50 servidores de Secretarías Técnicas y Oficinas de Recursos Humanos de distintas entidades públicas a nivel nacional, quienes intervienen en el desarrollo de los procedimientos administrativos disciplinarios.

\section{ARGUMENTACIÓN}

\section{Capacitación Recibida sobre Régimen Disciplinario}

Tal como se ha indicado en la parte introductoria del presente artículo, el procedimiento administrativo disciplinario regulado por la Ley $\mathrm{N}^{\circ} 30057$ - Ley del Servicio Civil, se encuentra sujeto a las garantías inherentes al debido procedimiento (derecho de defensa, deber de motivación, competencia de las autoridades, derecho a ser notificado, entre otros), así como a los principios del procedimiento administrativo sancionador (legalidad, tipicidad, non bis in ídem, razonabilidad, impulso de oficio, verdad material, presunción de licitud, entre otros), previstos en el Texto Único Ordenado de la Ley $\mathrm{N}^{\circ} 27444$ - Ley del Procedimiento Administrativo General, aprobado por Decreto Supremo $\mathrm{N}^{\circ}$ 004-2019-JUS.

Bajo dicha premisa, cuando en el desarrollo del procedimiento administrativo disciplinario se transgrede alguna garantía inherente al debido procedimiento o algún principio del procedimiento administrativo sancionador, se produce un vicio que genera la declaración de nulidad del citado procedimiento. En esa línea, la capacitación sobre régimen disciplinario dirigida a los 
servidores públicos que intervienen en el desarrollo de los procedimientos administrativos disciplinarios es un elemento esencial y necesario para que estos eviten incurrir en la transgresión de las citadas garantías y principios, de modo que no se produzcan vicios de nulidad.

En concordancia con lo señalado, la Autoridad Nacional del Servicio Civil - SERVIR, en su condición de ente rector del Sistema Administrativo de Gestión de Recursos Humanos, a través de su Escuela Nacional de Administración Pública - ENAP, ha brindado capacitaciones sobre régimen disciplinario a diversos servidores públicos a nivel nacional. Sobre ello, de acuerdo con la información recabada mediante solicitud de acceso a la información pública, se tiene que durante el año 2019 la ENAP realizó 74 capacitaciones en la materia referida al régimen disciplinario, capacitando a un total de 4445 servidores públicos a nivel nacional, conforme fue informado mediante Oficio $\mathrm{N}^{\circ}$ 000334-2020-SERVIR-ACCESO A LA INFORMACIÓN. Dicha iniciativa si bien es positiva, aun no resulta suficiente considerando que de acuerdo al Informe sobre las características del servicio civil peruano, realizado por SERVIR (2016), solo al 2014 existían alrededor de "1'400,000.00 mil servidores públicos" (p. 3).

En ese contexto, habiéndose encuestado a servidores que laboran principalmente en las Secretarías Técnicas y en las Oficinas de Recursos
Humanos de 50 entidades, se ha obtenido el siguiente resultado en la Tabla 1 :

A partir de lo expuesto en la Tabla 1 se evidencia que el $62 \%$ (31), es decir, la mayor parte de las entidades donde laboran los servidores encuestados no ha recibido capacitación sobre régimen disciplinario en el año 2019 por parte de la Autoridad Nacional del Servicio Civil, así como también se observa que el 30\% (15) ha recibido entre 1 a 2 capacitaciones, lo que demuestra que la capacitación recibida es escasa llegando a ser incluso en la mayor parte nula o inexistente. En tanto que solo el 8\% (4) ha recibido entre 3 a 4 capacitaciones.

Asimismo, los datos contenidos en la Tabla 1 revelan que de las 31 entidades que recibieron 0 capacitaciones, 27 incurrieron en vicios de nulidad; de las 15 entidades que recibieron entre 1 a 2 capacitaciones, 12 incurrieron en vicios de nulidad; de las 4 entidades que recibieron entre 3 a 4 capacitaciones, 2 incurrieron en vicios de nulidad.

Lo precedentemente expuesto se muestra gráficamente en la Figura 1.

De esta manera, se aprecia que a menor capacitación recibida mayor es la incidencia de la producción de vicios de nulidad. Siguiendo este orden de ideas, la escasa capacitación recibida se pone de manifiesto e incide en los errores que

Tabla 1

Capacitaciones recibidas de SERVIR

\begin{tabular}{cccc}
\hline Número de Capacitaciones SERVIR & Entidades & Entidades & Número de PAD Nulos \\
\hline & & 4 & 0 \\
0 & 31 & 4 & $1-5$ \\
& & 8 & $6-15$ \\
& & 15 & $16-30$ \\
\hline & 15 & 3 & 0 \\
& & 5 & $1-5$ \\
& & 2 & $6-15$ \\
& & 3 & $16-30$ \\
& & 2 & 31 a más \\
\hline & & 2 & 0 \\
& 4 & 1 & $1-5$ \\
\hline
\end{tabular}

Nota. Elaboración propia 
Figura 1

Incidencia de la capacitación en la declaración de nulidad de los PAD

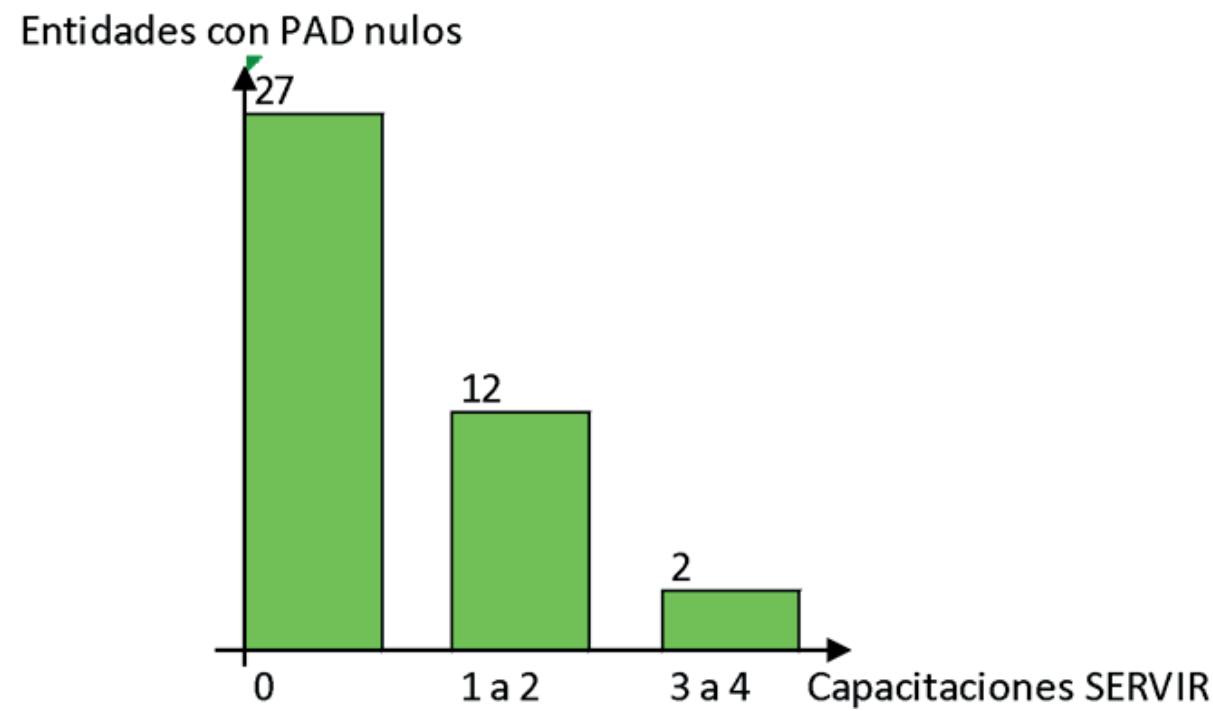

Nota. Elaboración propia

se cometen en la gestión de los procedimientos administrativos disciplinarios, generándose así vicios de nulidad que ocasionan la declaración de nulidad de los citados procedimientos.

Por consiguiente, se concluye que la escasa capacitación guarda relación con el alto índice de entidades que incurren en vicios de nulidad en la gestión de sus procedimientos administrativos disciplinarios en el año 2019.

\section{Prescripción de los Procedimientos Ad- ministrativos Disciplinarios}

El artículo 94 de la Ley № 30057 - Ley del Servicio Civil establece los plazos de prescripción para el ejercicio de la potestad disciplinaria de las entidades públicas, es decir, el plazo dentro del cual se debe iniciar los procedimientos administrativos disciplinarios, así como el plazo para imponer la sanción, cuyo vencimiento ocasiona la pérdida de dicha potestad. Así, se tiene el plazo de tres (3) años para el inicio del procedimiento contados a partir de la comisión de la falta y dentro de dicho plazo, el plazo de un (1) año desde la toma de conocimiento por parte de la Oficina de Recursos Humanos; asimismo, para la duración del procedimiento, esto es, desde el inicio hasta la imposición de la sanción, se establece el plazo de un (1) año.
Ahora bien, la declaración de nulidad de un procedimiento administrativo disciplinario implica que el mismo tenga que retrotraerse hasta el momento en que se produjo el vicio, el cual en ocasiones puede producirse al instaurarse el procedimiento o durante su desarrollo. En esa medida, el transcurso del tiempo que implica volver a desarrollar el procedimiento o volver a desarrollar una actuación determinada; ocasiona, al mismo tiempo, que también transcurran los plazos de prescripción tanto para el inicio del procedimiento como para la duración del mismo, según sea el caso. Atendiendo a lo señalado, en mérito a las encuestas practicadas, se ha obtenido el siguiente resultado en la Tabla 2:

A partir de lo expuesto en la Tabla 2 se advierte que 10 entidades tuvieron de 1 a 5 procedimientos administrativos disciplinarios declarados nulos, de los cuales prescribieron de 1 a 5 procedimientos en 7 entidades; en tanto que las 3 entidades restantes no tuvieron procedimientos prescritos. Asimismo, se aprecia que 11 entidades tuvieron de 6 a 15 procedimientos administrativos disciplinarios declarados nulos, de los cuales prescribieron de 6 a 15 procedimientos en 3 entidades y de 1 a 5 procedimientos en 5 entidades; en tanto que las 3 entidades restantes no tuvieron procedimientos prescritos. 
Tabla 2

Prescripción de los PAD declarados nulos

\begin{tabular}{cccc}
\hline Número de PAD nulos & Entidades & Entidades & Número de PAD prescritos \\
\hline 0 & 9 & 9 & 0 \\
\hline \multirow{2}{*}{$1-5$} & 10 & 3 & 0 \\
& & 7 & $1-5$ \\
\multirow{2}{*}{$6-15$} & \multirow{2}{*}{11} & 3 & 0 \\
& & 5 & $6-5$ \\
\hline \multirow{2}{*}{$16-30$} & 18 & 3 & $1-5$ \\
& & 7 & $6-15$ \\
\hline \multirow{2}{*}{31 a más } & 2 & 11 & $1-5$ \\
& & 1 & $6-15$ \\
\hline
\end{tabular}

Nota. Elaboración propia

Así también, se tiene que 18 entidades tuvieron de 16 a 30 procedimientos administrativos disciplinarios declarados nulos, de los cuales prescribieron de 6 a 15 procedimientos en 11 entidades y de 1 a 5 procedimientos en las 7 entidades restantes. De igual modo, se observa que 2 entidades tuvieron de 31 a más procedimientos administrativos disciplinarios declarados nulos, de los cuales prescribieron de 6 a 15 procedimientos en una de ellas, y de 1 a 5 procedimientos en la otra. Lo expuesto se muestra gráficamente en la siguiente Figura 2:

Sobre la base de lo expuesto en dicha Figura 2 , se advierte la relación existente entre la declaración de nulidad de los procedimientos administrativos disciplinarios y la prescripción de los mismos, puesto que de las 41 entidades que tuvieron procedimientos administrativos disciplinarios declarados nulos, 35 tuvieron procedimientos administrativos disciplinarios que prescribieron como consecuencia de dicha declaración de nulidad, es decir, el 85\%. De ese modo, se muestra que la declaración de nulidad de los procedimientos administrativos disciplinarios en el año 2019 incide en la prescripción de dichos procedimientos.

\section{Demandas de Indemnización}

Como marco referencial debe tenerse presente que, de conformidad con lo establecido en el numeral 95.2 del artículo 95 de la Ley $\mathrm{N}^{\circ}$ 30057 - Ley del Servicio Civil, la ejecución de las sanciones disciplinarias es inmediata, no se suspende por la interposición de recursos administrativos, sino que se ejecuta inmediatamente después de su notificación. Siendo así, las sanciones de suspensión sin goce de remuneraciones o destitución se ejecutan de manera inmediata impidiéndose al servidor público prestar servicios y, por ende, percibir sus remuneraciones. Sin embargo, cuando dichas sanciones - ya ejecutadas por completo o ejecutadas parcialmente - son declaradas nulas, los servidores interponen demandas de indemnización a fin que las entidades reparen el daño causado al haberles impedido prestar servicios y percibir sus remuneraciones. Al respecto, la Autoridad Nacional del Servicio Civil (2018), mediante el Informe Técnico $\mathrm{N}^{\circ}$ 1670-2018-SERVIR/GPGSC ha señalado lo siguiente:

\section{Conclusiones}

3.1 No procede el pago de remuneración por períodos no laborados como consecuencia de la imposición de una sanción de suspensión posteriormente declarada nula, toda vez que el pago de conceptos remunerativos se otorga como contraprestación por el trabajo efectivamente realizado. Sin perjuicio de ello, el servidor afectado, puede ejercer su derecho ante la instancia respectiva (vía proceso contencioso administrativo) a efectos de solicitar el pago de la Indemnización correspondiente. (p. 2)

Atendiendo a lo señalado, en mérito a las encuestas practicadas, se ha obtenido el siguiente resultado en la Tabla 3: 
A partir de lo expuesto en la Tabla 3 se advierte que de las 10 entidades que tuvieron de 1 a 5 procedimientos administrativos disciplinarios declarados nulos, 4 entidades tuvieron de 1 a 5 demandas de indemnización interpuestas por la ejecución de sanciones declaradas nulas; en tanto que las 6 entidades restantes no tuvieron demandas de indemnización. Asimismo, se aprecia que de las 11 entidades que tuvieron de 6 a 15 procedimientos administrativos disciplinarios declarados nulos, 7 entidades tuvieron de 1 a 5 demandas de indemnización interpuestas por la ejecución de sanciones declaradas nulas; en tanto que las 4 entidades restantes no tuvieron demandas de indemnización.

Así también, se tiene que de las 18 entidades que tuvieron de 16 a 30 procedimientos administrativos disciplinarios declarados nulos, 11 entidades tuvieron de 1 a 5 demandas de indemnización interpuestas por la ejecución de sanciones declaradas nulas; y 7 entidades tuvieron de 6 a 15 demandas de indemnización interpuestas por la ejecución de sanciones declaradas nulas. De igual modo, se observa que

Figura 2

Relación entre la declaración de nulidad de los PAD y la prescripción

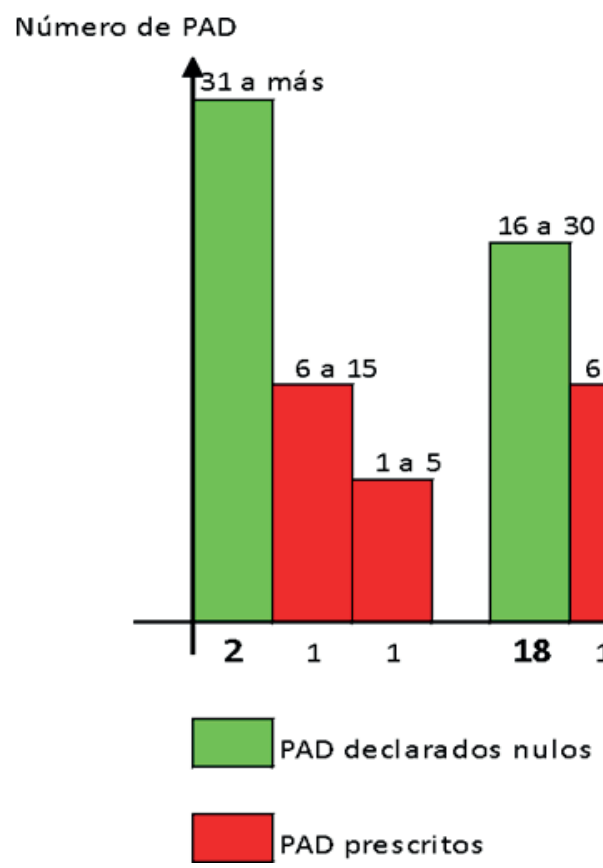

Nota. Elaboración propia

Tabla 3

Demandas de indemnización por las sanciones nulas

\begin{tabular}{|c|c|c|c|}
\hline Número de PAD nulos & Entidades & Entidades & Demandas de indemnización \\
\hline 0 & 9 & 9 & 0 \\
\hline \multirow{2}{*}{$1-5$} & \multirow{2}{*}{10} & 6 & 0 \\
\hline & & 4 & $1-5$ \\
\hline \multirow{2}{*}{$6-15$} & \multirow{2}{*}{11} & 4 & 0 \\
\hline & & 7 & $1-5$ \\
\hline \multirow{2}{*}{$16-30$} & \multirow{2}{*}{18} & 11 & $1-5$ \\
\hline & & 7 & $6-15$ \\
\hline \multirow{2}{*}{31 a más } & \multirow{2}{*}{2} & 1 & 0 \\
\hline & & 1 & $1-5$ \\
\hline
\end{tabular}

Nota. Elaboración propia 
de las 2 entidades que tuvieron de 31 a más procedimientos administrativos disciplinarios declarados nulos, 1 entidad tuvo de 1 a 5 demandas de indemnización interpuestas por la ejecución de sanciones declaradas nulas; en tanto que la entidad restante no tuvo demandas de indemnización. Lo expuesto se muestra gráficamente en la siguiente Figura 3:

Sobre la base de lo expuesto en la Figura 3 , se advierte que de las 41 entidades que tuvieron procedimientos administrativos disciplinarios declarados nulos, 30 entidades tuvieron demandas de indemnización interpuestas en su contra por la ejecución de sanciones declaradas nulas, es decir, el 73\%. De ese modo, se muestra que la declaración de nulidad de los procedimientos administrativos disciplinarios en el año 2019 incide en la interposición de demandas de indemnización por la ejecución de sanciones declaradas nulas.

\section{CONCLUSIONES}

- La escasa capacitación que reciben los servidores que intervienen en el desarrollo de los procedimientos administrativos disciplinarios, principalmente los servidores de las Secretarías Técnicas y las Oficinas de Recursos Humanos, incide en la producción de vicios de nulidad. Si bien la Autoridad Nacional del Servicio Civil ha desplegado loables esfuerzos en capacitar sobre régimen disciplinario a los servidores públicos a nivel nacional, aún hace falta ampliar el alcance de dichas capacitaciones a más servidores.

- La declaración de nulidad de los procedimientos administrativos disciplinarios implica que los referidos procedimientos tengan que retrotraerse para volver a desarrollarse; lo que, al mismo tiempo, también genera que transcurran los plazos de prescripción de inicio y de duración del procedimiento. Por tanto, se concluye que la declaración de nulidad de los procedimientos administrativos disciplinarios incide en la prescripción de los mismos.

\section{Figura 3}

Relación entre la declaración de nulidad de los PAD y las demandas de indemnización por la ejecución de sanciones declaradas nulas

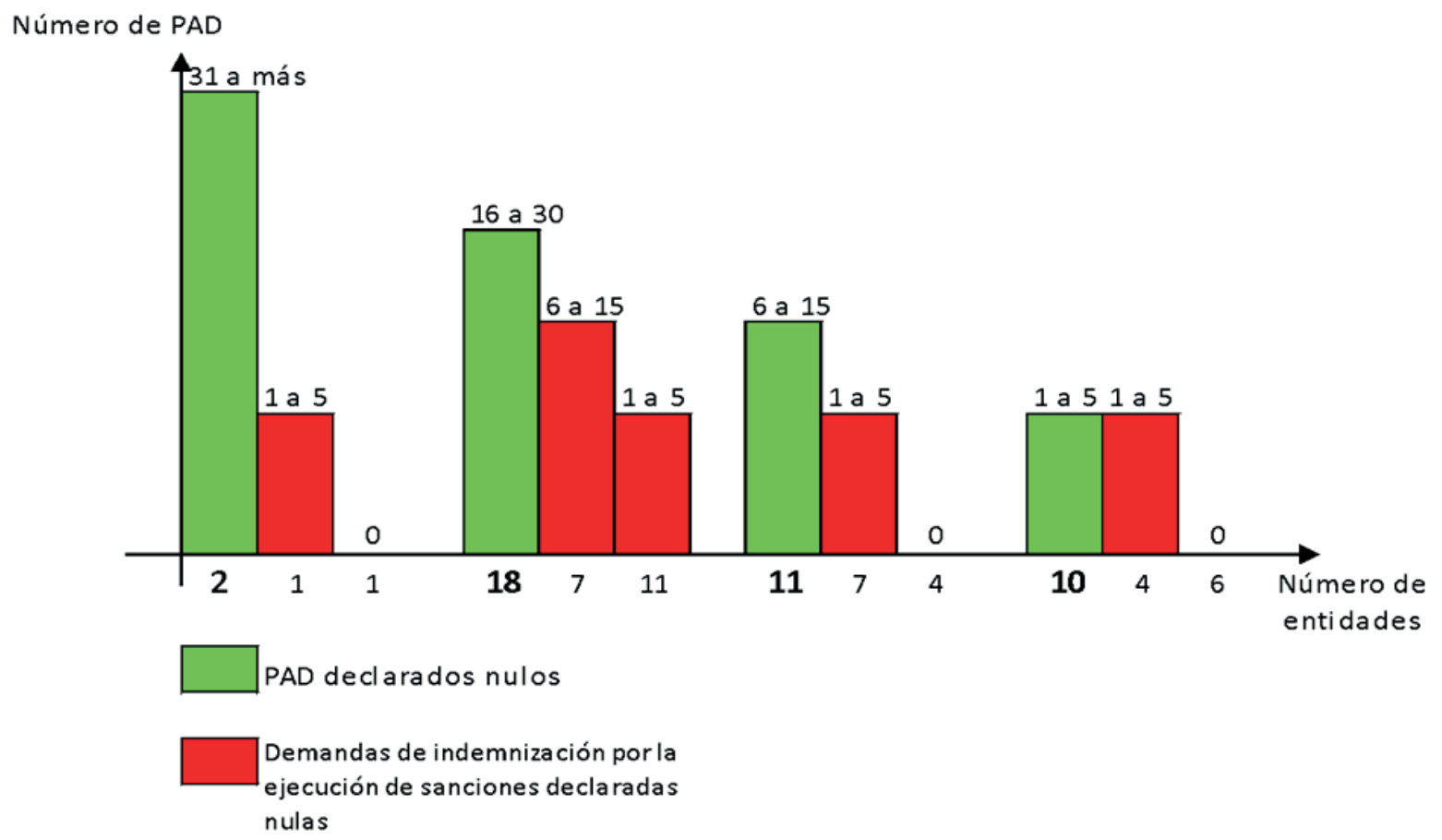

Nota. Elaboración propia 
- La declaración de nulidad de los procedimientos administrativos disciplinarios ocasiona que los servidores que acataron la ejecución de una sanción declarada nula, dejando de forma obligada de prestar servicios y de percibir sus remuneraciones; interpongan demandas de indemnización contra las entidades a efectos que se les repare el daño causado. Por tanto, se concluye que la declaración de nulidad de los procedimientos administrativos disciplinarios incide en la interposición de demandas de indemnización por la ejecución de sanciones declaradas nulas.

\section{REFERENCIAS BIBLIOGRÁFICAS}

Autoridad Nacional del Servicio Civil. (2016). Informe sobre las características del servicio civil peruano. https://cdn.www.gob.pe/uploads/document/file/1205615/SERVIR-El_servicio_civil_peruano-Anx1_feb2016.PDF

Autoridad Nacional del Servicio Civil. (2018). Informe Técnico $N^{\circ}$ 1670-2018-SERVIR/GPGSC. https://storage.servir.gob.pe/normatividad/ Informes_Legales/2018/IT_1670-2018-SERVIR-GPGSC.pdf

Autoridad Nacional del Servicio Civil. (2020). Oficio $\mathrm{N}^{\circ}$ 000334-2020-SERVIR-ACCESO A LA INFORMACIÓN.
Congreso de la República del Perú. (2013, 4 de julio). Ley № 30057. Ley del Servicio Civil. Diario Oficial El Peruano. https://leyes.congreso.gob.pe/ Documentos/Leyes/30057.pdf

Marina Jalvo, B. (2006). El Régimen Disciplinario de los Funcionarios Públicos. Editorial Lex Nova.

Morales Morante, C. (2020). Conversatorio con Carlos Guillermo Morales Morante. Revista Gestión Pública \& Control, (9), 56-60.

Presidencia de la República. (2019, 25 de enero). Decreto Supremo $\mathrm{N}^{\circ}$ 004-2019-JUS. Decreto Supremo que aprueba el Texto Único Ordenado de la Ley $N^{\circ}$ 27444, Ley del Procedimiento Administrativo General. Diario Oficial El Peruano. https://cdn.www.gob.pe/uploads/document/ file/320709/DS_004-2019-JUS.pdf

Presidencia de la República. (2014, 13 de junio). Decreto Supremo No 040-2014-PCM. Decreto Supremo que aprueba el Reglamento General de la Ley $N^{\circ} 30057$, Ley del Servicio Civil. Diario Oficial El Peruano. https://sgp.pcm.gob.pe/ wp-content/uploads/2015/06/DS-040-2014PCM.pdf

Tamayo, G. (2001). Diseños muestrales en la investigación. Revista Semestre económico, 4(7). https://dialnet.unirioja.es/servlet/articulo?codigo $=5262273$ 
